PERSPECTIVE

\title{
Toll-like receptors in ocular immunity and the immunopathogenesis of inflammatory eye disease
}

\author{
J H Chang, P J McCluskey, D Wakefield
}

Br J Ophthalmol 2006;90:103-108. doi: 10.1136/bjo.2005.072686

Microbial agents have an important role in the pathogenesis of various inflammatory eye diseases, such as uveitis and keratitis. Microbial infections of the eye such as microbial keratitis, ocular onchocerciasis, bacterial endophthalmitis, viral retinitis, and other infectious uveitis are unfortunately common. In addition, microbial agents have been implicated in the pathogenesis of "noninfectious" immune mediated diseases such as HLA-B27 associated acute anterior uveitis. Toll-like receptors (TLR) are a family of pattern recognition receptors that initiates rapid host innate immune response to microbial components known as pathogen associated molecular patterns, which are unique to a given class of microbes, such as lipopolysaccharide of Gram negative bacteria. Recent in vitro and in vivo studies have demonstrated the expression and function of TLRs in the eye, with significant implications for better understanding of ocular immunity and the pathogenesis of inflammatory eye diseases affecting the cornea, uvea, and retina.

See end of article for authors' affiliations

Correspondence to: Correspondence to:
Professor Denis Wakefield, School of Medical Sciences, University of New South Wales, Sydney, NSW 2052, Australia; d.wakefield@ unsw.edu.au

Accepted for publication 1 August 2005
I nflammatory eye diseases can affect any part of the eye from the ocular surface to the retina, the optic nerve, and other orbital structures. The consequences of inflammation in the eye, whether appropriate (as in the case of immune response to infective threats) or inappropriate (as in the case of autoimmune or allergic responses), may be sight threatening, as there is extreme sensitivity of this high fidelity system to even the slightest loss of clarity or to micro-anatomical distortions along the visual axis. Consequently, the eye normally exhibits "immune privilege" to avoid the potential sight destroying consequences of ocular inflammation. Ocular immune privilege involves certain anatomical, cellular, and soluble factors, such as the blood-ocular barrier and immunosuppressive factors of the aqueous humour. ${ }^{1}$ Despite these mechanisms to prevent potentially harmful ocular inflammation, such a state of immune privilege can break down, resulting in sight threatening inflammatory eye diseases such as uveitis and keratitis. Both infectious and immune mechanisms are important in triggering the breakdown in ocular immune privilege and in the development of various forms of inflammatory eye diseases. Microbial infections of the eye are unfortunately common, such as herpes simplex virus (HSV) keratitis and keratouveitis, Pseudomonas keratitis, ocular onchocerciasis, bacterial endophthalmitis, toxoplasmic retinochoroiditis, and cytomegalovirus (CMV) retinitis. Furthermore, it is becoming increasingly apparent that microbial agents may be important in the pathogenesis of "noninfectious" immune mediated inflammatory diseases, such as HLA-B27 associated acute anterior uveitis (AAU). ${ }^{2}$

A major advance in our understanding of infection and immunity occurred with the discovery of a new family of innate immune receptors, called the toll-like receptors (TLRs). TLRs enable the host immune system to recognise and respond to microbes by their "signature" molecular component(s), triggering the earliest immune responses that lead to inflammation. This review provides an overview of TLR research of specific relevance to the eye and provides a perspective on the implications of these findings for better understanding ocular immunity and the immunopathogenesis of inflammatory eye disease.

\section{TOLL-LIKE RECEPTORS AND THE IMMUNE RESPONSE}

There are two major arms to the immune response that detect and defend against microbial challenges or discriminate between self and non-self antigens. Innate immunity is the first line of host defence that is responsible for the immediate and rapid immune response to microbial challenge and has been considered, until recently, to be non-specific. In contrast, adaptive immunity is delayed, requiring clonal expansion, is antigen specific, mediated by antigen receptors on $\mathrm{B}$ and $\mathrm{T}$ lymphocytes, and is characterised by immunological memory. The innate immune system uses a variety of pattern recognition receptors (PRRs) including: those that are expressed on the cell surface, such as the phagocytic C-type lectin receptors and the transmembrane signalling TLRs; those that are expressed intracellularly, such as the nucleotide

Abbreviations: $A A U$, acute anterior uveitis; $A M D$, age related macular degeneration; $A P C$, antigen presenting cell; CMV, cytomegalovirus; DC, dendritic cell; dsRNA, double stranded RNA; EIU, endotoxin induced uveitis; HLA, human leucocyte antigen; HSV, herpes simplex virus; IFN- $\beta$, interferon $\beta$; IL, interleukin; KO, knockout; LPS, lipopolysaccharide; MHC, major histocompatibility complex; MIP-2, macrophage inflammatory protein-2; $N F-\kappa B$, nuclear factor $\kappa B$; NOD, nucleotide oligomerisation domain; PAMP, pathogen associated molecular patterns; PECAM-1, platelet endothelial cell adhesion molecule 1; POS, photoreceptor outer segments; PRR, pattern recognition receptor; RPE, retinal pigment epithelium; TIR, Toll/IL-1 receptor; Th, T helper; TLR, tolllike receptor; TNF- $\alpha$, tumour necrosis factor $\alpha$ 
oligomerisation domain (NOD) proteins; or secreted pattern recognition molecules, such as mannan binding lectins. TLRs have recently emerged as a key component of the innate immune system that detects microbial infection and triggers inflammation and antimicrobial host defence.

TLRs are a family of phylogenetically conserved PRRs of the innate immune system that recognise unique "molecular signature patterns" of microbial components, referred to as pathogen associated molecular patterns (PAMPs), although these are produced not only by pathogens but by all microorganisms. ${ }^{3}{ }^{4}$ Thus, PAMPs such as lipopolysaccharide (LPS) of Gram negative bacterial cell wall, are invariant among a given class of microbes and are produced by microbes but not by the host, and are therefore unique molecular signatures that confer a degree of specificity to innate immunity, facilitating self-non-self discrimination. There are at least 10 human TLRs identified to date. Each TLR has unique ligand specificity (table 1). For example, TLR4 recognises LPS of Gram negative bacteria; TLR2 recognises peptidoglycan from Gram positive bacteria; TLR3 recognises double stranded RNA (dsRNA) produced during virus replication; and TLR9 recognises CpG DNA of bacteria..$^{3-6}$ In addition to the recognition of exogenous, microbe derived PAMPs, there are reports of endogenous or "self" ligands for TLRs, such as the heat shock proteins and fibrinogen. However, the possibility that minute amounts of potential LPS contamination in such endogenous ligand preparations might be responsible for the observed TLR activation has not been excluded. ${ }^{4}$ Nevertheless, these reports of potential endogenous ligands for TLRs are intriguing and may have potential implications for understanding the pathogenesis of chronic inflammatory and autoimmune diseases.

Structurally, TLRs are type I transmembrane proteins with leucine rich repeats in the extracellular domain for ligand recognition, and Toll/IL-l receptor (TIR) domain in the cytoplasmic portion for intracellular signalling. ${ }^{5}$ TLRs are expressed on immune cells that are most likely to first encounter microbes, such as neutrophils, monocytes, macrophages, and dendritic cells (DCs). ${ }^{5}$ Ligand recognition by TLRs facilitates the dimerisation of TLRs that triggers the activation of signalling pathways, which originates from the cytoplasmic TIR domain, and culminates in the activation of the transcription factor, nuclear factor $\kappa \mathrm{B}(\mathrm{NF}-\kappa \mathrm{B})$, leading to the expression of pro-inflammatory genes such as TNF- $\alpha$, IL1 , and IL- $12 .{ }^{45}$ Innate recognition of PAMPs through TLR activation induces the expression of various pro-inflammatory cytokines, chemokines, adhesion molecules, and activates the effector functions of innate immune cells such as

Table 1 Summary of human toll-like receptors (TLRs) and their known ligands ${ }^{4}$

\begin{tabular}{|c|c|}
\hline TLR & Principal exogenous ligand(s) \\
\hline TLR2* & $\begin{array}{l}\text { Lipoproteins/lipopeptides (various pathogens)* } \\
\text { Peptidoglycan and lipoteichoic acid (Gram positive } \\
\text { bacteria) } \\
\text { Zymosan (fungi) }\end{array}$ \\
\hline TLR3 & Double stranded RNA (viruses) \\
\hline TLR4 & $\begin{array}{l}\text { LPS (Gram negative bacteria) } \\
\text { Bacterial HSP60 } \\
\text { Respiratory syncytial virus coat protein }\end{array}$ \\
\hline TLR5 & Flagellin (flagellated bacteria) \\
\hline TLR7 & Imidazoquinolone antiviral drug \\
\hline TLR8 & $\begin{array}{l}\text { Single stranded RNA (viruses) } \\
\text { Imidazoquinolone antiviral drug }\end{array}$ \\
\hline TLR9 & Unmethylated CpG motifs of bacterial DNA \\
\hline TLR10 & Unknown \\
\hline
\end{tabular}

*TLR2 forms heterodimers with TLR1 and TLR6: TLR1 associates with TLR2 to recognise tri-acyl lipopeptides; TLR6/TLR2 heterodimer recognises diacyl lipopeptides. phagocytosis, and thus initiates a rapid inflammatory response characterised by the recruitment of leucocytes to the site of infection to eliminate the invading pathogen. ${ }^{34}$

TLRs expressed on professional antigen presenting cells (APCs), such as DCs and macrophages, are a critical link between the innate and adaptive immunity. TLR mediated activation of DCs induces DC maturation, with the production of pro-inflammatory cytokines, upregulation of costimulatory and major histocompatibility complex (MHC) molecules, and thereby enhances the antigen presenting capacity of DCs. ${ }^{8} 9$ Thus, TLR stimulation of APCs leads to the activation and priming of antigen specific, naive $\mathrm{T}$ cells, triggering the adaptive arm of the immune response. Various factors influence the differentiation of T helper type l (Thl) versus Th2 responses, but DC derived cytokines present during the initial phase of $\mathrm{T}$ cell activation play the most important part in this process. ${ }^{8}{ }^{9}$ It has been shown that specific PAMPs that stimulate different TLRs, induce distinct patterns of cytokines resulting in a Thl/Th2 polarisation that is most appropriate for the pathogen. For example, activation of TLR4 or TLR9 in DCs induces production of IL-12, thereby skewing Th differentiation towards the Thl type..$^{\text {s } 9}$ Although indirect activation of DCs by inflammatory mediators alone was shown to be able to support $\mathrm{T}$ cell clonal expansion, it could not promote Th cell differentiation, for which the direct recognition of PAMPs by DCs appears to be of critical importance. ${ }^{9}$ Thus TLRs are important in both triggering and modulating the activation of the adaptive immune response. ${ }^{389}$

In addition to innate immune cells, an array of TLRs is expressed by epithelial cells at host/environment interfaces including that of the skin, gastrointestinal tract, respiratory tract, and the urogenital tract. ${ }^{5}{ }^{10}$ Strategic expression of TLRs at such host/environment interfaces appears to have an important role in the first line of defence against microbial invasions at these sites.

TLRs are implicated in several chronic inflammatory and immune mediated disorders by various potential mechanisms, including those in which infectious agents have been proposed in disease initiation, in which endogenous damage signals or self antigens may cause chronic inflammation in a TLR dependent manner, or where TLRs may be involved in the breakdown of immune tolerance. TLRs have been implicated in the pathogenesis of chronic inflammatory diseases such as inflammatory bowel disease, rheumatoid arthritis, psoriasis, and multiple sclerosis. ${ }^{46}$ It is of interest that these diseases are not uncommonly associated with various inflammatory eye diseases, such as uveitis and scleritis. The following sections will review recent studies that have investigated the expression and function of TLRs in the eye, and discuss these findings in the context of ocular immunity and their potential implications for the pathogenesis of inflammatory eye diseases.

\section{TOLL-LIKE RECEPTORS IN THE EYE: POTENTIAL ROLE IN INFLAMMATORY EYE DISEASE \\ Cornea}

The cornea forms an avascular and transparent window at the ocular surface that functions to maintain a clear visual axis as well as protect against the elements of the harsh external environment, including microbial threats. Analogous to the expression of TLRs found at other epithelial surfaces, ${ }^{5}{ }^{10}$ it might be expected that the cornea would also be similarly endowed with a pattern of TLRs to defend the integrity of the ocular surface. However, the cornea also subserves integral visual function, and activation of such TLRs in the cornea could result in inflammation, which might compromise corneal transparency and vision. This may be appropriate if it is in response to invading pathogens, such 
as Pseudomonas aeruginosa, allowing rapid immune response to eliminate the invading bacteria and thus preserve corneal integrity. However, TLR activation may be inappropriate and self destructive if it is in response to the non-pathogenic, normal commensal flora of the ocular surface. Recently, a number of studies have investigated the expression and function of TLRs in the human cornea (table 2), with some conflicting results.

Song et al reported the expression of functional cell surface TLR4 in cultured human corneal epithelial cells with production of pro-inflammatory cytokine and chemokine upon stimulation with Pseudomonas derived LPS. ${ }^{11}$ This may be important in the rapid initiation of the host's innate immune response and the recruitment of inflammatory cells to the cornea in Gram negative bacterial corneal infections, such as Pseudomonas keratitis. Similarly, the reported expression of functional TLR2 by cultured corneal epithelial cells, which produced pro-inflammatory cytokines, chemokines, and antimicrobial peptide in response to peptidoglycan of Staphylococcus aureus, may have a role in the pathogenesis of Gram positive bacterial keratitis. ${ }^{12}$ In contrast with these studies, Ueta et al found that human corneal epithelial cells expressed TLR2 and TLR4 intracellularly but not at the cell surface, and were incapable of responding to LPS from $P$ aeruginosa and to peptidoglycan from $S$ aureus, at the level of $\mathrm{NF}-\kappa \mathrm{B}$ transcription or the secretion of pro-inflammatory molecules. ${ }^{13} \mathrm{~A}$ more recent study by the same group has found that, in contrast with that observed for TLR2 and TLR4, human corneal epithelial cells expressed functional cell surface TLR3 (receptor for viral dsRNA) that was capable of responding to its ligand by the induction of pro-inflammatory cytokines and IFN- $\beta$ production. ${ }^{14}$ It has been proposed that ocular surface epithelial cell associated mucosal immune system may create an immunosilent condition for TLR mediated innate immunity to prevent unnecessary inflammatory responses to normal bacterial flora. ${ }^{13}$ As the corneal epithelial cells on the ocular surface are normally exposed to commensal bacterial flora residing in the conjunctival sac, they may possess a unique downregulatory mechanism to prevent inappropriate TLR mediated stimulation cascades. ${ }^{13}$ There may indeed be regional specialisations in the expression and function of TLRs. For example, normal intestinal epithelial cells, which are continuously exposed to gut commensal bacteria, express extremely low levels of TLR4 and no MD-2, a critical co-receptor of TLR4, and thus do not normally respond to LPS. ${ }^{15}{ }^{16}$ The strategic expression of TLR5 at the basal and wing cell layers but not at the apical layers of the stratified human corneal epithelium again suggests a potential mechanism by which the corneal epithelium may remain inactive in response to non-pathogenic bacteria at the apical surface, but able to trigger TLR mediated innate immune response once the epithelial barrier has been breached. ${ }^{17}$
Among the resident cells of the normal human cornea, keratocytes (stromal fibroblasts) appear to be the major cellular source of chemokines that mediate the selective recruitment of leucocytes from the vascular limbus to the avascular cornea. ${ }^{18}$ Cultured human corneal fibroblasts have been shown to express transcripts for TLR4, MD-2, and CDI4 genes in vitro, and LPS stimulation of these corneal fibroblasts induced the production of chemokines for neutrophils and monocytes and expression of adhesion molecule. ${ }^{19}$ Furthermore, activation of TLR4 was shown to be a critical step in the pathogenesis of endotoxin induced keratitis, which develops after the murine corneal epithelium is abraded and exposed to LPS. ${ }^{20}$ Endotoxin induced keratitis was found to directly result from extensive neutrophil infiltration into the corneal stroma that was mediated by the neutrophil chemoattractant, macrophage inflammatory protein (MIP)-2, in the corneal stroma, and platelet endothelial cell adhesion molecule (PECAM)-1 on the vascular endothelium. TLR4 may be of critical pathogenic importance, as the increased in vivo expression of PECAM-1 and MIP-2, and the subsequent development of endotoxin induced keratitis were shown to be regulated by TLR $4 .{ }^{20} \mathrm{C} 3 \mathrm{H} /$ HeJ mice, which does not have functional TLR4, ${ }^{21}$ developed only mild endotoxin induced keratitis with significantly fewer neutrophils and stromal oedema, compared to the keratitis that developed in the congenic $\mathrm{C} 3 \mathrm{H} / \mathrm{HeN}$ mice with functional TLR4. ${ }^{20}$

TLR4 has been also implicated to have a critical role in the inflammatory response associated with the pathogenesis of ocular onchocerciasis (river blindness) ${ }^{22}$ Onchocerca volvulus is a parasitic nematode transmitted by the Simulium blackfly that causes corneal inflammation, which can lead to blindness. In $O$ volvulus infected individuals, adult worms survive in subcutaneous nodules and release millions of microfiliariae that migrate to the anterior regions of the eye. It is the host response to degenerating worms that can result in sight threatening ocular inflammation..$^{23}$ Recent studies implicate the role of the endosymbiotic bacteria called Wolbachia, which infects and is present within the filarial nematode, and not the worm itself, as the major stimulus for the host's inflammatory response associated with onchocercal keratitis. ${ }^{22} 23$ Studies using $O$ volvulus extracts (containing Wolbachia) to induce keratitis in $\mathrm{C} 3 \mathrm{H} / \mathrm{HeJ}$ (non-functional TLR4) and C3H/HeN (functional TLR4) mice have shown that the endosymbiotic derived endotoxin-like activity of $O$ volvulus and its activation of TLR4 regulates $O$ volvulus keratitis in vivo by modulating chemokine and adhesion molecule expression in the cornea. ${ }^{22}$ It has thus been proposed that in infected individuals, the innate inflammatory response is likely to be initiated by the release of Wolbachia endotoxin-like molecules from dead and degenerating worms, which activate TLR4 on resident corneal epithelial cells and keratocytes. This would then trigger the

\begin{tabular}{|c|c|c|}
\hline Ocular tissue & Cell type & TLR $^{*}$ \\
\hline Cornea & $\begin{array}{l}\text { Corneal epithelium } \\
\text { Cultured corneal epithelial cells } \\
\text { Cultured corneal fibroblasts }\end{array}$ & $\begin{array}{l}\text { TLR1, 2†, 3, 4†, 5†, 6, 7, 9, } 10^{131417} 17 \\
\text { TLR1, 2†, 3†, 4†, 5†, 6, 9 } 9^{11-1417} \\
\text { TLR4 }\end{array}$ \\
\hline \multirow{3}{*}{ Uvea } & Whole bulbar conjunctiva & $\mathrm{TLR}^{28}$ \\
\hline & Resident stromal APCs & TLR4 $\dagger^{2831}$ \\
\hline & Cultured iris endothelial cells & TLR $4^{31}$ \\
\hline \multirow[t]{2}{*}{ Retina } & Whole retina & $\mathrm{TLR}^{28}$ \\
\hline & Cultured RPE cells & TLRI, $2 \dagger, 3 \dagger, 4 \dagger, 5,6,7,9,10^{35} 40$ \\
\hline Sclera & Whole sclera & TLR4 ${ }^{28}$ \\
\hline
\end{tabular}


production of chemokines and induction of adhesion molecules on the limbal vessels, leading to the recruitment of neutrophils and other inflammatory cells to the cornea. ${ }^{22}$

Recent studies using TLR2 KO, TLR9 KO, and MyD88 KO mice have further supported the in vivo functional importance of the activation of TLR/MyD88 pathway in the development of corneal inflammation. ${ }^{24}$ These studies examined the development of keratitis after stimulation of the cornea of TLR KO mice that had received a superficial corneal abrasion with the relevant TLR ligands. For example, CpG DNA (TLR9 ligand) was able to induce keratitis in wild type mice but not in the TLR9 KO mice. Endotoxin induced keratitis (via TLR4) could be normally induced in these TLR9 KO mice. Furthermore, the ligands for TLR2, TLR-4, or TLR-9 were not able to induce keratitis in mice that lacked the common adaptor protein, MyD88, required for TLR intracellular signalling. These findings demonstrate that the murine cornea has functional TLR2, TLR-4, and TLR-9, and that activation of these by their respective PAMPs induces the development of keratitis, in a MyD88 dependent manner, by the secretion of chemokines and the recruitment of neutrophils into the corneal stroma. ${ }^{24}$

\section{Uvea}

Uveitis is a heterogeneous group of diseases characterised by acute, recurrent, or chronic inflammation of the uvea, the middle vascular coat of the eye. It is the most common cause of intraocular inflammatory disease and an important cause of visual impairment in most populations. ${ }^{25}$ Uveitis may be infectious in aetiology or non-infectious, predominantly immune mediated. Microbes may invade the intraocular environment and cause various forms of infectious uveitis including, for example, bacterial and fungal endophthalmitis, viral uveitis such as HSV keratouveitis and CMV retinitis, and toxoplasmic retinochoroiditis. HLA-B27 associated AAU is by far the most common form of "non-infectious" immune mediated uveitis. ${ }^{25}$ Although the precise pathogenic mechanisms are unknown, HLA-B27 associated AAU develops in a genetically predisposed individual in whom environmental factors appear to be involved in the initiation of uveitis. Substantial clinical and experimental evidence implicates the role for microbial triggers, especially Gram negative enterobacteria such as certain species of Klebsiella, Salmonella, Shigella, and Yersinia, and Chlamydia trachomatis, in the pathogenesis of HLA-B27 associated AAU. ${ }^{2}$ Therefore, microbes or their PAMPs and their interaction with TLRs may be of critical importance in better understanding the pathogenesis of infectious uveitis and the role of microbial triggers in the development of immune mediated, "noninfectious" uveitis such as AAU.

In endotoxin induced uveitis (EIU), a well established animal model for human AAU, LPS of Gram negative bacterial cell wall, when injected at sites remote from the eye induces an AAU without significantly affecting other tissues. ${ }^{26}$ However, not all mouse strains develop EIU and, in particular, it has been well recognised that the $\mathrm{C} 3 \mathrm{H} / \mathrm{HeJ}$ mice do not develop uveitis in response to LPS while the congenic, $\mathrm{C} 3 \mathrm{H} / \mathrm{HeN}$ (LPS responsive) mice develop severe EIU. ${ }^{27}$ The specific genetic basis for this LPS hyporesponsiveness in $\mathrm{C} 3 \mathrm{H} /$ HeJ mice was recently identified as being the result of a point mutation within the coding region of the Tlr4 gene, which results in a functional disruption of TLR4 signalling, ${ }^{21}$ thus implicating a critical pathogenic role for LPS and TLR4 in the pathogenesis of EIU. These, together with the implicated role of Gram negative bacterial triggers in the development of human AAU, led us to investigate the expression of TLR4 in the human uvea.

We have demonstrated the in vivo expression of TLR4 and its associated LPS receptor complex by a network of resident
APCs, mostly HLA-DR+ DCs, within the normal human uvea. $^{28}$ A relatively rich network of TLR4+ APCs were observed in the iris root and ciliary body, compared to that in the rest of the iris or choroid. These TLR4+ APCs appeared to be strategically placed in perivascular and subepithelial locations within the uveal stroma and suggest that such uveal APCs endowed with the complete LPS receptor complex are optimally positioned to detect and respond to either blood borne or intraocular LPS of Gram negative bacteria. ${ }^{28}$ This is analogous to the situation in the cornea where TLRs appear in a strategic location immediately below the point of likely microbial invasion. A similar network of resident APCs exists in the mouse uvea, ${ }^{29}$ and we (unpublished data) and others ${ }^{30}$ have found TLR4 mRNA expression in mouse eyes. ${ }^{30}$ It is tempting to speculate that a similar pattern of TLR4 expression by APCs, to that observed in the human eye, may be observed in the murine uvea and that this may be of pathogenic importance in the development of EIU. This awaits further investigation, but to date, such studies have been limited by the availability of suitable reagents for murine TLRs.

A highly selective and specific pattern of TLR4 protein expression was observed in the human uvea, with the epithelial and endothelial cells of the normal human iris and ciliary body not expressing TLR4 and MD-2 proteins. We proposed that this may reflect the unique immunologically privileged nature of the eye. ${ }^{28}$ Recently, Brito et al confirmed our findings with the detection of TLR4+ cells in the uveal stroma that were "probably resident macrophages and DCs." ${ }^{\prime 31}$ In contrast with our findings, ${ }^{28}$ however, Brito et al also reported TLR4 protein staining on the non-pigmented epithelial cells of the ciliary body and in the superficial layers of the retina. ${ }^{31}$ We had also observed a similar pattern of staining when using paraffin embedded ocular sections (which were not observed in our studies using fresh frozen sections ${ }^{28}$ ) and these were considered to be non-specific, as we observed an identical pattern of positive staining using the isotype matched negative control antibody (unpublished data).

Although the human iris endothelium does not express TLR4 protein in vivo, cultured human iris endothelial cells were found to express TLR4 mRNA and respond to LPS stimulation with the production of pro-inflammatory cytokines. ${ }^{31}$ This may have implications for understanding the apparent susceptibility of the anterior uvea to the breakdown of the blood-aqueous barrier and development of uveitis. Importantly, Brito et al showed that TLR4 in the human uvea can be functional, by demonstrating TLR4 dependent proinflammatory cytokine production in response to in vitro LPS stimulation of cultured uveal tissue explants. ${ }^{31}$ We had previously proposed that the preferential expression of TLR4 by the APCs within the uvea may suggest a novel mechanism for the initiating factors in the pathogenesis of AAU, which has a particular predilection for affecting this middle vascular layer of the eye. ${ }^{28}$ LPS derived from Gram negative bacteria may trigger AAU by activating TLR4 on uveal APCs that are optimally positioned in perivascular locations to activate leucocyte recruitment, from the vasculature to the uvea, by activating the secretion of chemokines and the expression of vascular adhesion molecules.

Mutations in the gene encoding NOD2 protein (a cytosolic PRR that detects intracellular bacteria) can result in Blau syndrome, characterised by a clinical triad of chronic granulomatous uveitis, arthritis, and dermatitis..2 Furthermore, mutations in the NOD2 gene are clearly implicated in the genetic predisposition to granulomatous bowel inflammation of Crohn's disease. ${ }^{33}$ A growing body of studies has found that functional polymorphisms in TLR genes such as TLR4 and TLR2, may affect the development 
and progression of a variety of human diseases. ${ }^{6}$ It would be important to investigate whether such TLR polymorphisms may be important genetic susceptibility factors in the development and/or progression of inflammatory eye diseases such as uveitis.

\section{Retina}

There appears to be a particular predilection for the involvement of the retina by various infectious agents, such as viruses (CMV and HSV retinitis) and the obligate intracellular protozoan, Toxoplasma gondii, the commonest cause of posterior uveitis. ${ }^{25}$ Retinal pigment epithelium (RPE) is a monolayer of cells strategically located between the neurosensory retina and the vascular choroid, forming part of the blood-retina barrier, and have been implicated to have a role in the immunopathogenesis of uveoretinitis. ${ }^{34}$ Recent studies have begun to explore the role of TLRs in retinal innate immunity (table 2 ).

Cultured human RPE cells expressed the genes for TLRs 17, TLR-9, and TLR-10, with the highest expression level found for TLR3 ${ }^{35}$ TLR3 is the receptor for dsRNA produced by most viruses during their replication. ${ }^{4}$ Human RPE stimulation with a synthetic dsRNA was able to induce the secretion of IFN- $\beta$ in a TLR3 dependent manner, and these RPE cells were in turn sensitive to the antiviral action of IFN- $\beta$. Furthermore, TLR3 stimulation of RPE cells induced the secretion of various cytokines, chemokines, and adhesion molecule. ${ }^{35}$ Thus, TLR3 mediated signalling, triggered by dsRNA of virus replication, may have a protective role in viral infections of the retina. RPE cells appear to be one of the principal cellular targets for infectious agents, such as CMV and Toxoplasma gondii. ${ }^{36}{ }^{37}$ TLRs have been implicated in innate immune recognition of $T$ gondii. In particular, TLR2 appears to have an important role in the innate immune response to $T$ gondii, although additional TLRs and ligands have also been implicated. ${ }^{38} 39$ The role of TLRs in the pathogenesis of toxoplasmic retinochoroiditis awaits further investigation.

RPE cells mediate the physiological phagocytosis of shed photoreceptor outer segments (POS) and thus the maintenance of outer retina homeostasis. TLR4 expressed on the cell surface of cultured human RPE cells have been reported to participate in the cellular handling of human POS. ${ }^{40}$ Human POS was shown to induce clustering of TLR4 at their cell surface binding sites and TLR4 may be one component necessary for the initiation of cellular signalling and the generation of reactive oxygen metabolites by human RPE cells in response to POS binding. ${ }^{40}$ From these findings it has been speculated that TLR4 clustering may also have a role in pro-inflammatory pathophysiological changes that accompany retinal diseases such as uveitis, age related macular degeneration (AMD), and proliferative vitreoretinopathy, as POS binding to RPE may create a susceptible, local proinflammatory environment in the retina. ${ }^{40} \mathrm{~A}$ recent study has reported a significant association between TLR4 polymorphism and AMD, with the demonstration of an increased risk of AMD in carriers of the D299G variant in the TLR4 gene. ${ }^{41}$

Given that RPE has the capacity to have a major immunological role at the blood-retina barrier and that it is a frequent site of involvement by various infectious agents of the retina, these early studies demonstrating the expression and function of TLRs by RPE cells implicate a potential role for these innate immune receptors in the pathogenesis of various inflammatory retinal diseases. RPE cells endowed with TLRs may be able to rapidly respond to infectious agents and limit the damage to the neurosensory retina. However, the resultant cellular activation may come at the expense of breakdown in the blood-retina barrier resulting from the production of various inflammatory mediators, leading to the development of potentially sight threatening retinochoroiditis.

\section{CONCLUSIONS}

The Toll rush has begun and we are only just beginning to explore the potential role of TLRs in ocular immunity and inflammation, and undoubtedly there will be many more studies to come in this exciting and promising area of research. TLRs are likely to have wide implications for ocular immunology, not only in inflammatory eye diseases but also in other areas such as corneal transplantation and intraocular tumours. The initial molecular mechanisms that lead to the loss of the normally sight protective state of ocular immune privilege and the development of various forms of ocular inflammation are currently poorly understood. Microbial agents or their PAMPs, via their interaction with TLRs and other PRRs, may be critically important in the pathogenesis of both infectious and, at least some forms of, non-infectious inflammatory eye diseases. A better understanding of these mechanisms is of fundamental importance in progressing our knowledge of ocular infection and immunity but also would be of major clinical significance, as it may identify potential new therapeutic targets that may be more selective, effective, and safer than the currently available therapies for treating this group of sight threatening inflammatory eye diseases.

\section{ACKNOWLEDGEMENTS}

JHC is supported by NH\&MRC of Australia (grant 222928).

\section{Authors' affiliations}

J H Chang, P J McCluskey, D Wakefield, Laboratory of Ocular Immunology, Inflammatory Diseases Research Unit, School of Medical Sciences, University of New South Wales, Sydney, Australia

J H Chang, P J McCluskey, D Wakefield, Department of Ophthalmology, St Vincent's Hospital, Sydney, Australia

P J McCluskey, Department of Ophthalmology, Royal Prince Alfred Hospital, Sydney, Australia

The authors have no competing interests regarding any aspects of this work.

\section{REFERENCES}

1 Streilein JW, Stein-Streilein J. Does innate immune privilege exist? J Leukoc Biol 2000;67:479-87

2 Chang JH, McCluskey PJ, Wakefield D. Acute anterior uveitis and HLA-B27. Surv Ophthalmol 2005; 50:364-88.

3 Iwasaki A, Medzhitov R. Toll-like receptor control of the adaptive immune responses. Nat Immunol 2004;5:987-95.

4 Takeda K, Akira S. Toll-like receptors in innate immunity. Int Immunol 2005:17:1-14.

5 Takeda K, Kaisho T, Akira S. Toll-like receptors. Annu Rev Immunol 2003;21:335-76.

6 Andreakos E, Foxwell B, Feldmann M. Is targeting Toll-like receptors and their signaling pathway a useful therapeutic approach to modulating cytokinedriven inflammation? Immunol Rev 2004;202:250-65.

7 Tsan MF, Gao B. Endogenous ligands of Toll-like receptors. J Leukoc Biol 2004;76:514-19

8 Mazzoni A, Segal DM. Controlling the Toll road to dendritic cell polarization. $J$ Leukoc Biol 2004;75:721-30.

9 Sporri R, Reis e Sousa C. Inflammatory mediators are insufficient for full dendritic cell activation and promote expansion of CD4+ T cell populations lacking helper function. Nat Immunol 2005;6:163-70.

10 Backhed F, Hornef M. Toll-like receptor 4-mediated signaling by epithelial surfaces: necessity or threat? Microbes Infect 2003;5:951-9.

11 Song PI, Abraham TA, Park Y, et al. The expression of functional LPS receptor proteins CDI 4 and toll-like receptor 4 in human corneal cells. Invest Ophthalmol Vis Sci 2001;42:2867-77.

12 Kumar A, Zhang J, Yu FS. Innate immune response of corneal epithelial cells to Staphylococcus aureus infection: role of peptidoglycan in stimulating proinflammatory cytokine secretion. Invest Ophthalmol Vis Sci 2004;45:3513-22.

13 Ueta M, Nochi T, Jang MH, et al. Intracellularly expressed TLR2s and TLR4s contribution to an immunosilent environment at the ocular mucosal epithelium. J Immunol 2004;173:3337-47.

14 Ueta M, Hamuro J, Kiyono $\mathrm{H}$, et al. Triggering of TLR3 by polyl: $\mathrm{C}$ in human corneal epithelial cells to induce inflammatory cytokines. Biochem Biophys Res Commun 2005;331:285-94. 
15 Abreu MT, Vora P, Faure E, et al. Decreased expression of Toll-like receptor-4 and MD-2 correlates with intestinal epithelial cell protection agains dysregulated proinflammatory gene expression in response to bacterial lipopolysaccharide. J Immunol 2001;167:1609-16.

16 Abreu MT Arnold ET, Thomas LS, et al. TLR4 and MD-2 expression is regulated by immune-mediated signals in human intestinal epithelial cells. J Biol Chem 2002;277:20431-7.

17 Zhang J, Xu K, Ambati B, et al. Toll-like receptor 5-mediated corneal epithelial inflammatory responses to Pseudomonas aeruginosa flagellin. Invest Ophthalmol Vis Sci 2003:44:4247-54.

18 Tran MT, Tellaetxe-Isusi M, Elner V, et al. Proinflammatory cytokines induce RANTES and MCP-1 synthesis in human corneal keratocytes but not in corneal epithelial cells. Beta-chemokine synthesis in corneal cells. Invest Ophthalmol Vis Sci 1996:37:987-96.

19 Kumagai N, Fukuda K, Fujitsu Y, et al. Lipopolysaccharide-induced expression of intercellular adhesion molecule-1 and chemokines in cultured human corneal fibroblasts. Invest Ophthalmol Vis Sci 2005;46:1 14-20.

20 Khatri S, Lass JH, Heinzel FP, et al. Regulation of endotoxin-induced keratitis by PECAM-1, MIP-2, and toll-like receptor 4. Invest Ophthalmol Vis Sci 2002;43:2278-84.

21 Poltorak A, He X, Smirnova I, et al. Defective LPS signaling in $\mathrm{C} 3 \mathrm{H} / \mathrm{HeJ}$ and C57BL/10ScCr mice: mutations in Tlr4 gene. Science 1998;282:2085-8.

22 Saint Andre A, Blackwell NM, Hall LR, et al. The role of endosymbiotic Wolbachia bacteria in the pathogenesis of river blindness. Science 2002;295:1892-5.

23 Hall LR, Pearlman E. Pathogenesis of onchocercal keratitis (river blindness). Clin Microbiol Rev 1999;12:445-53.

24 Johnson AC, Heinzel FP, Diaconu E, et al. Activation of toll-like receptor (TLR)2, TLR4, and TLR9 in the mammalian cornea induces MyD88-dependent corneal inflammation. Invest Ophthalmol Vis Sci 2005;46:589-595.

25 Chang JH, Wakefield D. Uveitis: a global perspective. Ocul Immunol Inflamm 2002;10:263-79.

26 Rosenbaum JT, McDevitt HO, Guss RB, et al. Endotoxin-induced uveitis in rats as a model for human disease. Nature 1980;286:611-13.

27 Li Q, Peng B, Whitcup SM, et al. Endotoxin induced uveitis in the mouse: susceptibility and genetic control. Exp Eye Res 1995;61:629-32.

28 Chang JH, McCluskey P, Wakefield D. Expression of toll-like receptor 4 and its associated lipopolysaccharide receptor complex by resident antigen- presenting cells in the human uvea. Invest Ophthalmol Vis Sci 2004;45:1871-8

29 McMenamin PG, Crewe J, Morrison S, et al. Immunomorphologic studies of macrophages and MHC class II-positive dendritic cells in the iris and ciliary body of the rat, mouse, and human eye. Invest Ophthalmol Vis Sci 1994; $35: 3234-50$

30 Tuaillon N, Shen de F, Berger RB, et al. MCP-1 expression in endotoxininduced uveitis. Invest Ophthalmol Vis Sci 2002;43:1493-8

31 Brito BE, Zamora DO, Bonnah RA, et al. Toll-like receptor 4 and CDIL expression in human ciliary body and TLR- 4 in human iris endothelial cells. Exp Eye Res 2004;79:203-8.

32 Rosenbaum JT, Planck SR, Davey MP, et al. With a mere nod, uveitis enters a new era. Am J Ophthalmol 2003;136:729-32.

33 Ogura $\mathrm{Y}$, Bonen DK, Inohara N, et al. A frameshift mutation in NOD2 associated with susceptibility to Crohn's disease. Nature 2001:411:603-6.

34 Holtkamp GM, Kijlstra A, Peek R, et al. Retinal pigment epithelium-immune system interactions: cytokine production and cytokine-induced changes. Prog Retin Eye Res 2001;20:29-48.

35 Kumar MV, Nagineni CN, Chin MS, et al. Innate immunity in the retina: Tolllike receptor (TLR) signaling in human retinal pigment epithelial cells. J Neuroimmunol 2004;153:7-15.

36 Detrick B, Rhame J, Wang Y, et al. Cytomegalovirus replication in human retinal pigment epithelial cells. Altered expression of viral early proteins. Invest Ophthalmol Vis Sci 1996:37:814-25.

37 Nagineni CN, Pardhasaradhi K, Martins MC, et al. Mechanisms of interferoninduced inhibition of Toxoplasma gondii replication in human retinal pigment epithelial cells. Infect Immun 1996;64:4188-96.

38 Scanga CA, Aliberti J, Jankovic D, et al. Cutting edge: MyD88 is required for resistance to Toxoplasma gondii infection and regulates parasite-induced IL12 production by dendritic cells. J Immunol 2002;168:5997-6001.

39 Mun HS, Aosai F, Norose K, et al. TLR2 as an essential molecule for protective immunity against Toxoplasma gondii infection. Int Immunol 2003;15:1081-7.

40 Kindzelskii AL, Elner VM, Elner SG, et al. Toll-like receptor 4 (TLR4) of retinal pigment epithelial cells participates in transmembrane signaling in response to photoreceptor outer segments. J Gen Physiol 2004;124:139-49.

41 Zareparsi S, Buraczynska M, Branham KEH, et al. Toll-like receptor 4 variant D299G is associated with susceptibility to age-related macular degeneration. Hum Mol Genet 2005; 14:1449-55. 